\title{
BMJ Open Effect of screening young men for Chlamydia trachomatis on the rates among women: a network modelling study for high-prevalence communities
}

\author{
Zhuolin Qu (D) , ${ }^{1,2}$ Asma Azizi, ${ }^{3}$ Norine Schmidt,, ${ }^{4}$ Megan Clare Craig-Kuhn, ${ }^{4}$ \\ Charles Stoecker, ${ }^{5}$ James Mac Hyman, ${ }^{1}$ Patricia J Kissinger (iD ${ }^{4}$
}

To cite: Qu Z, Azizi A, Schmidt N, et al. Effect of screening young men for Chlamydia trachomatis on the rates among women: a network modelling study for high-prevalence communities. BMJ Open 2021;11:e040789. doi:10.1136/ bmjopen-2020-040789

- Prepublication history and additional material is published online only. To view please visit the journal online (http://dx.doi. org/10.1136/bmjopen-2020040789).

Received 22 May 2020 Revised 23 December 2020 Accepted 05 January 2021

Check for updates

(C) Author(s) (or their employer(s)) 2021. Re-use permitted under CC BY-NC. No commercial re-use. See rights and permissions. Published by BMJ.

For numbered affiliations see end of article.

Correspondence to

Professor Patricia J Kissinger; kissing@tulane.edu

\section{ABSTRACT}

Objective Chlamydia trachomatis (Ct) is the most commonly reported sexually transmitted infection in the USA and causes important reproductive morbidity in women. The Centers for Disease Control and Prevention recommend routine screening of sexually active women under age 25 but not among men. Despite three decades of screening women, chlamydia prevalence in women remains high. Untested and untreated men can serve as a reservoir of infection in women, and male-screening based intervention can be an effective strategy to reduce infection in women. We assessed the impact of screening men on the Ct prevalence in women.

Design We created an individual-based network model to simulate a realistic chlamydia epidemic on sexual contact networks for a synthetic population $(n=5000)$. The model is calibrated to the ongoing routine screening among African American (AA) women in the USA and detailed a malescreening programme, Check It, that bundles best practices for Ct control. We used sensitivity analysis to quantify the relative importance of each intervention component.

Setting Community-based venues in New Orleans, Louisiana, USA.

Participants Heterosexual AA men, aged 15 to 24, who had sex with women in the past 2 months.

Intervention Venue-based screening, expedited index treatment, expedited partner treatment and rescreening. Results We estimate that by annually screening $7.5 \%$ of the AA male population in the age-range, the chlamydia prevalence would be reduced relatively by $8.1 \%(95 \% \mathrm{CI} 5.9 \%$ to $10.4 \%)$ in $\mathrm{AA}$ women and $8.8 \%(95 \% \mathrm{Cl} 6.9 \%$ to $10.8 \%)$ in $\mathrm{AA}$ men. Each man screened could prevent $0.062(95 \% \mathrm{Cl} 0.030$ to $0.094)$ cases in men and 0.204 (95\% $\mathrm{Cl} 0.143$ to 0.267$)$ cases in women. The model suggested the importance of intervention components ranked from high to low as venuebased screening, expedited index treatment, expedited partner treatment and rescreening.

Conclusion The findings indicated that male-screening has the potential to substantially reduce the prevalence among women in high-prevalence communities.

\section{INTRODUCTION}

Chlamydia trachomatis $(\mathrm{Ct})$ is the most commonly reported infectious disease in the USA, with over 1.7 million cases each year. ${ }^{1}$ It is a major cause of infertility, pelvic inflammatory disease
Strengths and limitations of this study

- The network-based model captures the complex assortative mixing in sexual partnerships among an African American (AA) population in New Orleans using the survey data on sexual behaviours.

- We present a novel modelling study to assess the impact of a new male-screening programme that bundles the best practices for chlamydia control on the current chlamydia prevalence in AA women, given the ongoing routine screening among $A A$ women in the USA.

- The proposed stochastic, heterosexual and individual-based model provided a flexible framework for a public health team to answer 'what if' questions that are hard to address in the field.

- The model is parametrised using data from two surveys on sexual behaviours, which reflects our best understanding of the AA population in the New Orleans area.

- The model assumed a closed and stable population in an area, and the quantitative results are only valid for a short-term prediction that does not consider impacts by the external factors, such as behaviour changes, natural disasters and changes in mixing patterns.

and ectopic pregnancy among women ${ }^{2}$ and has been associated with increased HIV acquisition. ${ }^{3}$ Because women experience the most severe sequelae, the focus of Ct prevention in the USA has been on screening sexually active women $<25$ years old, providing her and her partner(s) with treatment and rescreening. Despite three decades of screening women, chlamydia prevalence remains high in the sexually active young women in the USA. ${ }^{1}$

There is no recommendation for Ct screening among men in the USA. In 2007, an expert panel at the Centers for Disease Control and Prevention (CDC) concluded that the evidence is not sufficient to recommend routine screening for Ct in sexually active young men. ${ }^{4}$ The conclusion 
was based on the Ct prevalence in 2007 and the feasibility, efficacy and cost-effectiveness of screening men. However, since then, evidence of the benefit of screening young men for $\mathrm{Ct}$ in high prevalence areas has been mounting. More recent modelling studies indicate that screening men in high prevalence populations can be cost-effective due to averted cases among women. ${ }^{56}$

The community-based programme, Check It, ${ }^{7} \mathrm{Ct}$ screens African American (AA) men aged 15 to 24 in New Orleans. The core of this intervention is Ct screening for men. We hypothesise that men are an important reservoir of infection for women and therefore need to be targeted for intervention. ${ }^{7}$ The Check It programme bundles several key Ct control strategies (table 1):

- Venue-based screening (VBS) of participants at nonclinical community venues, such as barbershops, colleges and universities, in high-prevalence neighbourhoods characterised by similar demographic and geographical factors. This venue-based enrolment is enhanced with marketing strategies, such as the distribution of flyers, web education, social media and informational cards.

- Expedited treatment by providing medication for the Ct-positive men (or index) (expedited index treatment or EIT) and his sexual partner(s) (expedited partner treatment or EPT) via partnering community pharmacies without a medical examination to speed up the treatment of his sexual partners and reduce reinfection rates in the index.

- Rescreening of Ct-positive men; retesting for infection 3 months after treatment.

- Social network peer referral (SNPR) that encourages men to refer young AA men in their social network to Check It via flyers, social media or text messages to promote the programme and increase the total enrolment.

Mathematical models create frameworks for understanding the underlying epidemiology of disease and help test the potential effectiveness of different approaches to bring the epidemic under control. Our modelling effort created a detailed simulation to model the specific practices implemented by the Check It programme. Most chlamydia simulations studies use differential equation-based compartmental models. These models are parametrised at the population level and assume homogeneous mixing of the individuals in the same compartment. We formulated an agent-based model at a more granular level that captures the complex assortative mixing pattern in sexual partnership networks. The structure of the sexual network affects the spread of the infection and the effectiveness of the mitigation efforts. We calibrated the agentbased model based on the sexual behaviour surveys ${ }^{78}$ and assessed the impact of these male-screening-based strategies on mitigating the Ct epidemic among AA women aged 15 to 24 years old in New Orleans.

\section{METHODS}

We modelled the Ct transmission among a synthetic population connected through heterosexual partnership networks. These sexual networks were generated based on the data from two survey studies ${ }^{78}$ that investigated the sexual behaviour of young AA men and women in New Orleans. The model parameterised the transmission pathways on the individual level, and it simulated the sexual behaviour and kept track of the infection status for each individual in the synthetic population over time. We then modelled the intervention strategies, including both the standard preventive healthcare for women (routine Ct screening) and the Check It intervention, for each individual to study the impact of male-screening on the Ct prevalence in women.

\section{Generation of a synthetic population over dynamic sexual networks}

We constructed a closed 5000-member population, where the heterosexual partnerships were represented by bipartite sexual networks. The heterosexual networks captured the assortative mixing pattern among our targeted AA population by matching the population-level quantities (the degree distribution and joint-degree distribution, online supplemental appendix A.1) from two surveys: the ongoing Check It study ${ }^{7}$ and the 'You Geaux Girl!' (YGG) study, ${ }^{8}$ which enrolled 1318 AA men (May 2017 to April 2019, ongoing, age range 15 to 24) and 473 AA women (September 2012 to December 2015, completed, age range 18 to 19), respectively, in New Orleans. All

\begin{tabular}{ll}
\hline Table 1 & Summary of interventions involved in the Check It programme \\
\hline Intervention & Description \\
\hline VBS & $\begin{array}{l}\text { Venue-based screening by recruiting male participants at non-clinical community venues } \\
\text { EIT }\end{array}$ \\
EPT & $\begin{array}{l}\text { Expedited index treatment by providing medication to the Ct-positive men } \\
\text { examination }\end{array}$ \\
SNPR & $\begin{array}{l}\text { Social network peer referral encourages men to refer young AA men in their social network to Check It to } \\
\text { increase the total enrolment }\end{array}$ \\
Rescreening & Ct-positive men are retested for infection 3 months after treatment
\end{tabular}

AA, African American; Ct, Chlamydia trachomatis. 
participants gave written informed consent before taking part in the studies.

We assumed that the population is closed and men and women in our model only have sexual partnerships within this population cohort. This assumption closely agrees with our data sets, where about $92 \%$ of the male participants (Check It study) and $94 \%$ of the female participants (YGG study) have partners in the same age cohort (15 to 24 years old). The surveys also found that about $90 \%$ of the partners for male participants (Check It study) and 95\% of the partners for female participants (YGG study) were also AA. We included the technical details of network descriptions in online supplemental appendix A.1.

To further characterise sexual behaviours, we categorised one's partner as either primary or casual partner, depending on the survey responses to questions such as level of commitment and the duration of the relationship (detailed criteria in online supplemental appendix A.1.2 and the distributions in online supplemental appendix A.2). These categorisations can be asymmetric: A is B's primary partner, but B may be A's casual partner, and there are three types of partnerships in the networks: primaryprimary, primary-casual and casual-casual partnership.

When modelling the epidemics over a long time (several months or years), we updated the sexual partner(s) every 2 months (the time-frame covered by the Check It survey) and created a series of dynamic sexual networks that are evolving in time. To simulate the partner updating behaviour, we employed a detailed realistic social contact network that modelled the daily activities for 150000 people in New Orleans. ${ }^{9}$ Then the heterosexual networks were updated through people's contacts in the social network. Specifically, we assumed that the primary-primary partnerships were preserved throughout the simulation period and half of the primary-casual and all of the casual-casual partnerships are replaced by one's social contacts every 2 months (details in online supplemental appendix A.1.3). There were, on average, $80 \%$ of the sexual partnerships coming from one's heterosexual social network, which is in agreement with the Check It data $(76 \%$ of partners were reported as social contacts from school, work, the neighbourhood and so on).

\section{Chlamydia epidemic on dynamic sexual networks}

We modelled the infection status of each individual using the Susceptible-Infectious-Susceptible (SIS) framework. All uninfected individuals are susceptible to being infected, and all infected people recover to this susceptible state after either spontaneous recovery or treatment. A susceptible person can be infected by his/her infectious partner. The force of infection is the probability (per day) that a susceptible person will be infected. This probability was estimated by considering risk factors of how many partners the person has, the type of partnership with each partner, the probability of having sexual contact per partner per day (contact rate) and the probability of using a condom.
We estimated the sexual contact rates for the primary and casual partnerships from the data sets (see table A.3). We observed that the contact rate was higher for primary partnerships than for casual partnerships, and there was a decreasing trend in the per partner contact rate when the number of partners increases. Moreover, the data sets gave a higher probability of using a condom with a casual partner than with a primary one (table 2 ). We introduced a condom failure rate to include cases when the condom is not used properly.

The details on the configuration of the epidemic model over networks are fully described in (online supplemental appendix A.2).

\section{Modelling the intervention strategies}

Our goal was to investigate the net impact of screening men through the Check It programme given the existing screening policy for women and the ongoing endemic Ct epidemic. To this end, we outlined the intervention strategies for both women and men in separate flow charts in figure 1. The baseline scenario accounts for Ct screenings completed at women's annual exams as part of regular preventive healthcare. We also included the Ct screenings prompted by symptomatic infections (clinical visits) in the baseline scenario for both men and women.

\section{Existing intervention strategies}

The current (baseline) Ct mitigation efforts for women (the right side of figure 1) include Ct screening during routine annual exams and clinical visits for symptomatic infections. The model assumes that the same fraction $\left(\sigma_{a}^{w}\right.$ ) of women return for a physical exam each year (more details in online supplemental appendix A.3). Symptoms can appear in a small fraction of infected women $\left(\sigma_{s}^{w}\right)$, and we assumed that a fraction $\theta_{s}$ of these women get medical care within an average of $\tau_{s}$ days, including incubation period and appointment scheduling process, after infection. ${ }^{10}$ After the diagnostic test, we assumed that all the positive cases get index treatment in an average of $\tau_{t}^{w}$ days. Moreover, the CDC recommends EPT for infected women by providing treatment to the patient to bring to her partner(s) without first examining the partner(s). ${ }^{11}$ We assumed that a fraction $\theta_{p}^{w}$ of the partners are treated with an average delay of $\tau_{p}^{w}$ days. The EPT fraction $\theta_{p}^{w}$ is the product of (1) the fraction of the physicians practising EPT as recommended and (2) the fraction of compliance from the notified partners. Last, women diagnosed with Ct infection should be retested after the initial treatment. ${ }^{12}$ Thus, we assumed a fraction $\theta_{r}^{w}$ of treated women are retested for infection $\tau_{r}^{w}$ days after the initial treatment.

Since routine male-screening is not recommended and is rarely practised, ${ }^{13}$ we only account for screenings prompted by symptomatic infections. Similar to the process in women, we assumed that there is a small fraction $\left(\sigma_{s}^{m}\right)$ of infected men that develop symptomatic Ct infections, and, with a delay of $\tau_{s}$ days, a fraction $\theta_{s}$ of them get screening and treatment. Moreover, follow-up 
Table 2 Model parameters for the AA population aged 15-24

\begin{tabular}{|c|c|c|c|c|}
\hline & Description & Baseline & $\begin{array}{l}\text { Range for } 95 \% \\
\text { Confidence } \\
\text { Interval }\end{array}$ & Reference \\
\hline$\beta^{m 2 w}$ & Transmissibility from men to women per contact & 0.30 & 0.04 to $0.5^{\star}$ & 1516 \\
\hline$\beta^{w 2 m}$ & Transmissibility from women to men per contact & 0.10 & 0.04 to 0.25 & 1516 \\
\hline$\tau_{n}$ & Average time to recovery without treatment (years) & 1.32 & exponential & 18 \\
\hline$c_{p}$ & Fraction of condom use for primary partners & 0.54 & - & $\begin{array}{l}\text { online supplemental } \\
\text { appendix A.6.1 }\end{array}$ \\
\hline$c_{c}$ & Fraction of condom use for casual partners & 0.66 & - & $\begin{array}{l}\text { online supplemental } \\
\text { appendix A.6.1 }\end{array}$ \\
\hline$c_{\epsilon}$ & Condom failure rate & 0.1 & - & 22 \\
\hline$\sigma_{a}^{w}$ & Fraction of the target women who are screened annually & 0.6 & 0.56 to 0.65 & 2425 \\
\hline$\sigma_{s}^{w}$ & Fraction of symptomatic infection in women & 0.3 & - & 10 \\
\hline$\theta_{p}^{w}$ & Fraction of partner treatment for index women & 0.24 & - & Derived \\
\hline & - Fraction of physicians practicing partner treatment & 0.4 & 0.3 to 0.5 & 26 \\
\hline & - Fraction of compliance for partner treatment & 0.6 & 0.4 to 0.8 & 2728 \\
\hline$\theta_{r}^{w}$ & Fraction of treated women who are rescreened & 0.2 & 0.17 to 0.28 & 29 \\
\hline$\tau_{t}^{w}$ & Time lag in treatment for screened women (days) & 2 & - & 30 \\
\hline$\tau_{p}^{w}$ & Time lag in partner treatment for treated women (days) & 6 & 0 to 15 & 27 \\
\hline & - Fraction of SNPR enrolment & 0.24 & & $\begin{array}{l}\text { online supplemental } \\
\text { appendix A.6.2 }\end{array}$ \\
\hline$\rho$ & Number of peer-recruited men per VBS-enrolled man & 0.32 & & Derived \\
\hline$\sigma_{s}^{m}$ & Fraction of symptomatic Ct infection in men & 0.11 & & 10 \\
\hline$\theta_{t}^{m}$ & Fraction of screened positive men treated (EIT) & 0.76 & 0.1 to 0.9 & $\begin{array}{l}\text { online supplemental } \\
\text { appendix A.6.3 }\end{array}$ \\
\hline$\theta_{p}^{m}$ & Fraction of partner treatment for index men (EPT) & 0.27 & 0.1 to 0.9 & $\begin{array}{l}\text { online supplemental } \\
\text { appendix A.6.3 }\end{array}$ \\
\hline$\theta_{r}^{m}$ & Fraction of treated men with rescreening & 0.12 & 0.1 to 0.9 & $\begin{array}{l}\text { online supplemental } \\
\text { appendix A.6.3 }\end{array}$ \\
\hline$\tau_{t}^{m}$ & Time lag in treatment for screened men (days) & 12 & - & $\begin{array}{l}\text { online supplemental } \\
\text { appendix A.6.3 }\end{array}$ \\
\hline$\tau_{n}^{m}$ & Time lag in screening for men enrolled via SNPR (days) & 7 & - & Assume \\
\hline$\tau_{p}^{m}$ & Time lag in partner treatment for treated men (days) & 2 & - & $\begin{array}{l}\text { online supplemental } \\
\text { appendix A.6.3 }\end{array}$ \\
\hline$\tau_{r}^{m}$ & Time lag in rescreening for treated men (days) & 102 & - & $\begin{array}{l}\text { online supplemental } \\
\text { appendix A.6.3 }\end{array}$ \\
\hline
\end{tabular}

Continued 
*For the disease transmissibility parameters $\left(\beta^{m 2 w}\right.$ and $\left.\beta^{w 2 m}\right)$, the estimated ranges come from the mathematical modelling papers. In our study, we estimated the baseline values for these transmission rates by calibrating the model to the Ct prevalences in the AA population within the age range (10.2\% in men and $13.5 \%$ in women).

AA, African American; Ct, Chlamydia trachomatis; EIT, expedited index treatment; EPT, expedited partner treatment; SNPR, social network peer referral; VBS, venue-based screening.

interventions, such as partner treatment or rescreening after the index treatment, are not commonly implemented in clinics for men. ${ }^{13}$ Therefore, follow-up interventions for men were not included in the model.

\section{Check It intervention strategies}

The Check It programme recruited participants in community venues, including community colleges, historically black colleges and universities, barbershops and other community-based organisations. This venue-based enrolment is enhanced with marketing strategies (distribution of flyers, web education, social media and informational cards) and enrols a fraction $\sigma_{e}^{m}$ of the target male population for Ct screenings.

Some participants learnt about the programme through their social networks, such as text messages and information cards sent by friends or word-of-mouth. We accounted for this peer impact as SNPR and included it as a source of enrolment (see table A.4). On average, the proportion between the non-peer enrolled men and peer-recruited men is $1: \rho$, and we assumed that peer-referred men are enrolled in the programme with an average delay of $\tau_{n}^{m}$ days from the time the referring man enrolled.

We modelled the non-peer enrolment process as a random sampling from the entire male population. Meanwhile, we modelled the peer-referred enrolment (SNPR) by searching the background social network of each non-peer enrolled man and randomly sampling among the eligible candidates.

The rest of the intervention practice was modelled similarly to women's cases: a fraction $\theta_{t}^{m}$ of the screened and infected men receive EIT after a delay of $\tau_{t}^{m}$ days. A fraction $\theta_{p}^{m}$ of these men's partners receive EPT with a delay of $\tau_{p}^{m}$ days. Finally, a fraction $\theta_{r}^{m}$ of the index men return for rescreening $\tau_{r}^{m}$ days after the initial infection.
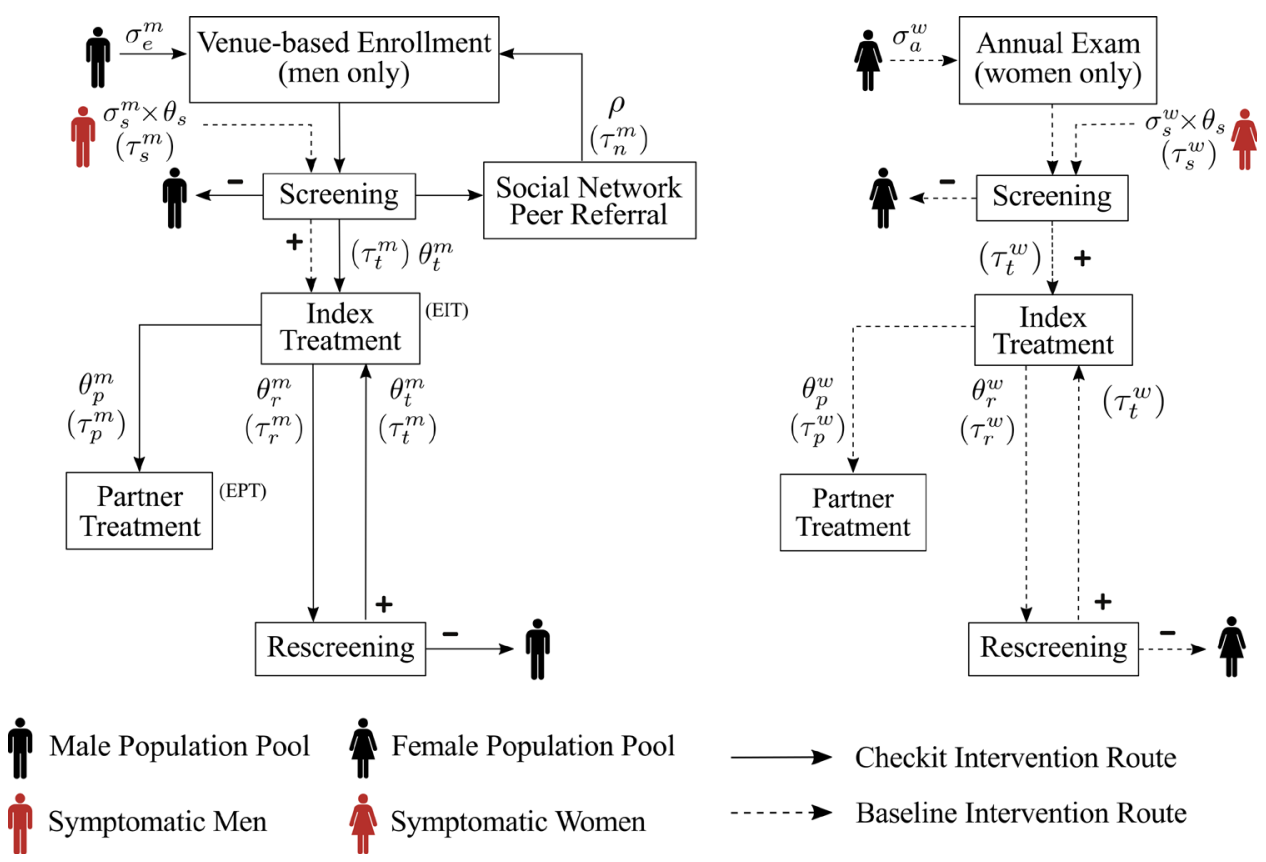

Figure 1 Flowchart for Ct intervention strategies in men and women. The solid lines are the new practices incorporated in the male-screening programme, Check It, and the dashed lines are the existing interventions implemented in the healthcare system. These current interventions include women's annual screening and screenings prompted by symptomatic infections in both men and women. Our modelling effort assessed the net impact of the male-screening programme to help control the Ct epidemic. The Check It programme targets the male population and uses venue-based enrolment, expedited index treatment, expedited partner treatment, rescreening and social network peer referral (see table 1). The intervention parameters are marked along the routes indicating the rates of compliance and delays, obtained from either literature or Check It data (see table 2). Ct,Chlamydia trachomatis. 


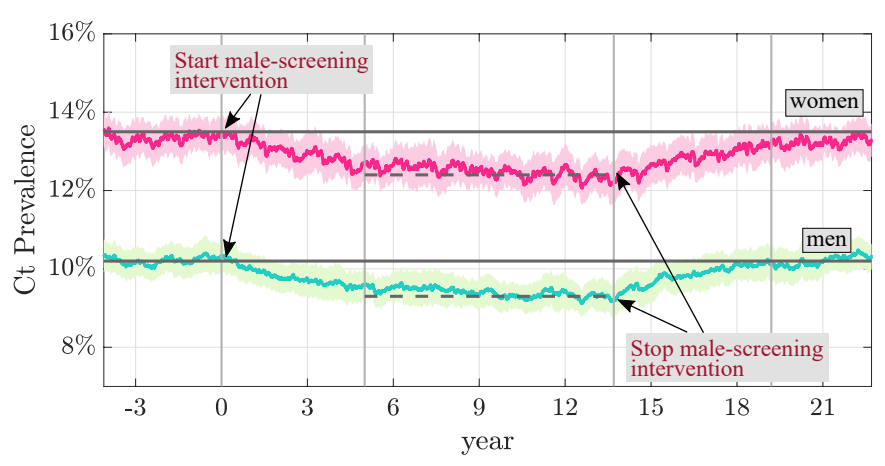

Figure 2 Impact of male-screening programme implemented at the existing intervention level. The curves are the mean of 50 stochastic simulations, and the bands around the curves indicate the one SD. The baseline Ct prevalences (before year 0) are $13.5 \%$ and $10.2 \%$ in women and men, respectively. At year 0 , the male-screening intervention is turned on. Around year 5, the Ct prevalences reach quasisteady states, which are $12.4 \%$ in women and $9.3 \%$ in men. The prevalences are reduced by $8.1 \%$ in women and $8.8 \%$ in men relatively. When the male-screening programme is stopped around year 14 , the Ct prevalences return to the baseline levels in about 5.5 years. Ct,Chlamydia trachomatis.

If the rescreened men are infected with Ct, then they are treated as index cases, and the intervention process is repeated. Details on the estimates of compliance rates and delays were summarised in table A.5 and A.6.

\section{Model parameters and calibration}

The model parameters used in the simulations (table 2) represent our best knowledge of the current Ct epidemic and mitigation efforts among the AA population in the age range 15 to 24 in the USA. The values for the parameter Ct transmission probabilities per sexual contact between men and women $\left(\beta^{m 2 w}\right.$ and $\left.\beta^{w 2 m}\right)$ are not clear in the current biological literature. Quinn et $_{a l} \mathrm{l}^{14}$ gives the estimate of infection frequency per partnership. There are modelling studies ${ }^{15} 16$ that provide rough estimates with large variations on the probability of transmission per contact. Because of the large uncertainty with these two parameters, they may vary in a wide range and are treated as tuning parameters for model calibration.

We calibrated the model to fit the current Ct prevalence in New Orleans among the AA population aged 15 to 24 (10.2\% in men and $13.5 \%$ in women). ${ }^{17}$ This prevalence reflects both the high infection rate in the region and the ongoing mitigation efforts among women. To have a realistic initial infection within the sexual network, we set the initial infection population to be consistent with the distribution of an emerging epidemic. We obtained such a quasi-steady-state balanced initial condition by starting a small epidemic in the past and letting it grow to the current (pre-Check It) Ct endemic state (online supplemental appendix A.4). This initialisation process considered the existing (baseline) combination of $\mathrm{Ct}$ interventions (dashed routes in figure 1) to give a comprehensive approximation of the current Ct control before the launch of Check It.

Approximately half of the Ct infections in women are cleared naturally by the first year after being infected and $80 \%$ are cleared after 2 years. ${ }^{18}$ We fitted an exponential distribution for the average time of natural recovery $\tau_{n}$ days to the data from, ${ }^{18}$ and we assumed the same distribution for men and women. Our numerical simulations suggest that the model results are not sensitive to this assumption (see Discussion and online supplemental appendix A.5). We also assumed the recovery time with treatment also follows an exponential distribution $\tau_{t} \sim \exp (1 / 7)$ in days.

\section{Sensitivity analysis}

The model parameters in table 2 represent the best-guess estimates for practical scenarios, and we used local and extended sensitivity analysis to quantify the most significant model parameters. ${ }^{19}$ To check the impact of each component of the Check It intervention bundle one-ata-time, we conducted the extended sensitivity analysis by varying each intervention parameter (the parameter of interest), while fixing the other parameters. We then checked its corresponding impact on the Ct prevalence for women, men and the overall population (the quantities of interest).

For the local sensitivity analysis, we defined the relative local sensitivity index of a quantity of interest, q, with respect to the parameter of interest, $\mathrm{p}$, as $S_{p}^{q}=p / q \times \partial q / \partial p$ . This normalised sensitivity index, $S_{p}^{q}$, measures the percentage change in an output quantity given the percentage change in an input parameter. If an input parameter, p, changes by $x \%$, then the output quantity, $\mathrm{q}$, changes by $S_{p}^{q} \times x \%$.

To further investigate the synergistic effect of the intervention components beyond the current levels, which could be limited by the protocols and available resources of Check It, we conducted the global sensitivity analysis by varying two intervention parameters together while fixing all other parameters. We then predicted the impact under different combinations of intervention parameters.

\section{Patient and public involvement}

There was no patients or public involvement in designing, conducting, reporting on or dissemination of information related to our modelling study.

\section{RESULTS}

Impact of male-screening programme at existing intervention level

We quantified the impact of the male-screening programme implemented at the current level of intervention intensity (as shown in table 2). Figure 2 shows the change in Ct prevalences after the launch of the malescreening intervention for men at year 0 with a balanced initial condition (online supplemental appendix A.4). Around year 5 of the programme, the Ct prevalences are controlled at much lower levels (quasi-steady states): 

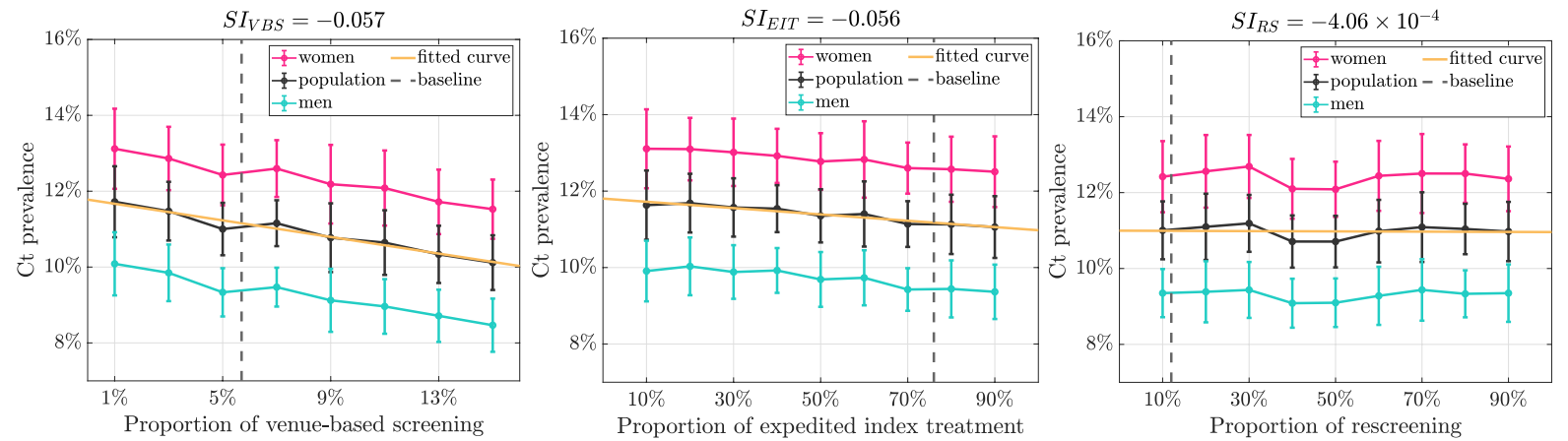

Figure 3 Local and extended sensitivity analysis on Check It intervention parameters (on the $\mathrm{x}$-axis) against the Ct prevalence (y-axis). For each plot, the parameter of interest is varied while the other model parameters are fixed as in table 2. The Ct prevalences for men, women and the entire population are plotted, which are averaged over the time-frame year 4.5 5.5 of 30 simulations. The error bars give the one SD above and below the average. The local sensitivity indices (q=quantity of interest is Ct prevalence) are given in the titles. Ct,Chlamydia trachomatis; EIT,expedited index treatment; VBS, venue-based screening.

\section{$12.4 \%(95 \%$ CI $12.1 \%$ to $12.7 \%)$ in women and $9.3 \%$} $(95 \%$ CI $9.1 \%$ to $9.5 \%)$ in men, which are reduced relatively by $8.1 \%$ ( $95 \%$ CI $5.9 \%$ to $10.4 \%$ ) in women and $8.8 \%$ (95\% CI $6.9 \%$ to $10.8 \%$ ) in men. When stopping the male-screening programme later around year 14, the Ct prevalences return back to the baseline scenario in about 5.5 years.

In fact, near the lower quasi-steady states, our model predicts the following annual statistics from the programme based on a population of 5000:

- Each year, the programme conducts in total 174 screenings, including 42 from peer-recruited participants (SNPR), and achieves 13 treatments for index men (EIT) and 8 treatments for partners of those men (EPT).

- Among all the screened men found to be Ct-positive, the average number of partners within the past 2 months is 2.30 (95\% CI 2.25 to 2.35 ).

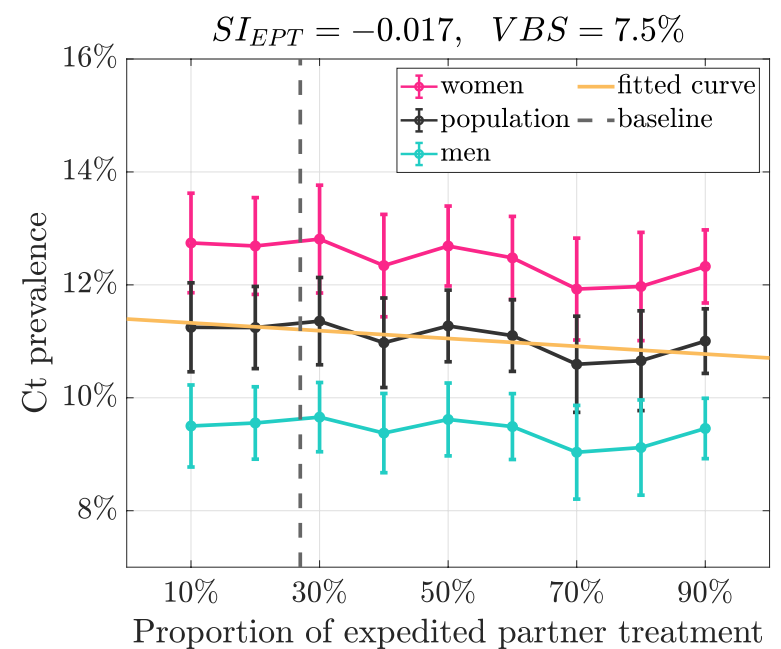

- Compared with the scenario without Check It, the programme prevents 10.8 (95\% CI 5.3 to 16.3) cases in men and 35.6 (95\% CI 24.8 to 46.4$)$ cases in women per year.

- Roughly, for each man screened, it could prevent 0.062 (95\% CI 0.030 to 0.094 ) cases in men and 0.204 (95\% CI 0.143 to 0.267$)$ cases in women.

\section{Significance of the components of the intervention}

The sensitivity analysis quantified the relative significance of the intervention components in the programme. In the results presented below, we have considered the quantity of interest to be Ct prevalence and omit the upper index in the sensitivity index for the simplicity of the presentation.

From figures 3 and 4 (left), the Ct prevalences have an almost linear response to the intervention parameters, and the sensitivity at the current level of Check It intensity

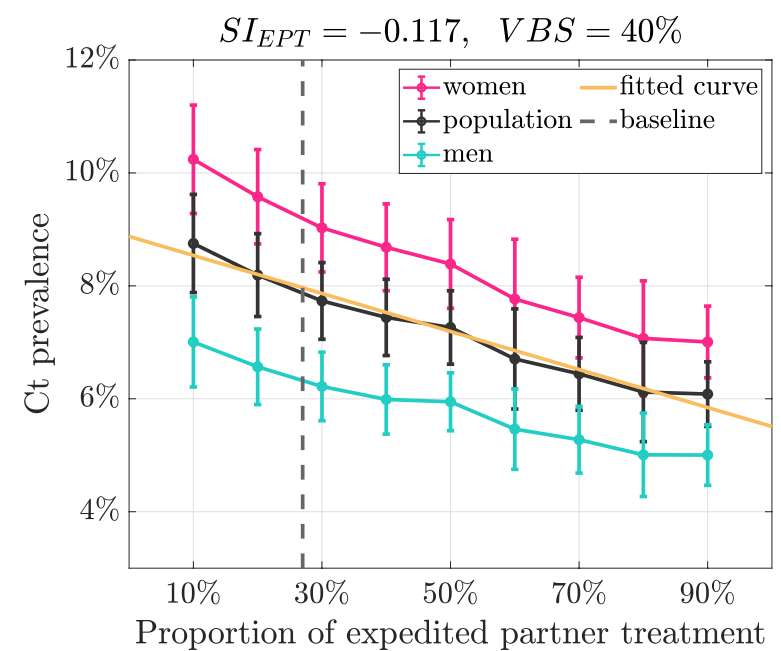

Figure 4 Local and extended sensitivity analysis on expedited partner treatment (on the $x$-axis) against the Ct prevalence (y-axis). Left: the analysis at the current level of Check It intervention intensity, screening 7.5\% of the target male population. Together with the results in figure 3 and at the current Check It level, the significance of intervention components is ranked as venue-based screening $\approx$ expedited index treatment >expedited partner treatment >rescreening. Right: the analysis at a much higher $40 \%$ male-screening rate while fixing other intervention parameters. The magnitude of the local sensitivity index is almost seven times larger (-0.117 vs -0.017$)$, which suggests that the partner treatment becomes more important in reducing prevalence when increasing the screening coverage in men. Ct,Chlamydia trachomatis; EIT,expedited index treatment. 


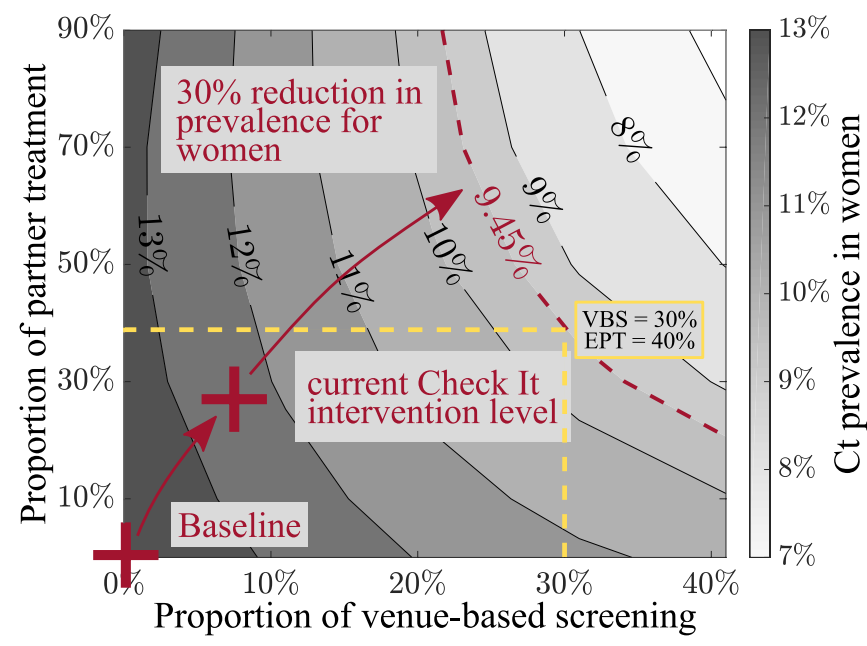

Figure 5 Global sensitivity analysis of Ct prevalence in women (marked in contour lines) against two intervention parameters: venue-based screening (VBS, x-axis) and expedited partner treatment (EPT, $y$-axis) on a uniform $5 x$ 5 grid. At each grid point, the Ct prevalence is averaged over the time-frame year 4.5 5.5 of 10 simulations, and the contour surface is smoothed by a least-square fit of a two-dimensional quadratic polynomial to the grid values. The baseline scenario (VBS=0\% and EPT $=0 \%$ ) and current intervention level (VBS $=7.5 \%$ and $\mathrm{EPT}=27 \%$ ) are marked in crosses, which shows an $8.1 \%$ relative reduction in women's prevalence. To achieve a $30 \%$ relative reduction in women's Ct prevalence, the combined intervention levels required are marked by the dashed contour line. For example, with a coverage of $30 \%$ male-screening and $40 \%$ partner treatment, the model predicts that the $\mathrm{Ct}$ prevalence in $\mathrm{AA}$ women will be reduced to $9.45 \%$. AA,African American; Ct, Chlamydia trachomatis.

is ranked from high to low as VBS, EIT, EPT, rescreening, where the sensitivity of VBS is close to EIT. Moreover, when increasing the coverage of VBS from $7.5 \%$ to $40 \%$ (figure 4), the magnitude of the corresponding sensitivity index for EPT becomes seven times larger $(-0.117$ vs -0.017), which suggests that EPT would be much more effective in reducing the prevalence with high malescreening coverage.

We then conducted a global sensitivity analysis using the two most significant parameters from the local and extended sensitivity analysis: VBS and EPT. The response plot for women's Ct prevalence (figure 5) shows that the male-screening strategy has the potential to reduce the Ct prevalence in women substantially, and it predicts the effectiveness under different combinations of intervention intensities. For example, the model estimates the combination of VBS $=30 \%$ of the target men and $\mathrm{EPT}=40 \%$ of their partners will give a $30 \%$ reduction in Ct prevalence among women after 5 years of intervention.

\section{DISCUSSION}

Our model provides a framework for public health workers to ask 'what if' questions that are hard to evaluate in the field. We simulated the Ct epidemic over sexual networks based on a young AA population in New Orleans, where the ongoing female-screening interventions are not sufficient to bring down the high Ct prevalence in women. From the simulation results at the current intervention level (figure 2), by annually screening men in the target population (AA, sexually active, aged 15 to 24 ), together with other best practices in Ct control, there is the potential to further mitigate Ct prevalence from the baseline (female-screening intervention only) among this highprevalence cohort. Moreover, once the male-screening programme is suspended, the prediction shows that the prevalence will return to the baseline level given no significant changes in the underlying force of infection in the population. This suggests that, similar to the existing recommendation of annual screening policy for high-risk women, the male-screening intervention also requires long-term efforts to maintain its effectiveness over time. With the joint force of the interventions for both men and women, Ct could be controlled at much lower prevalences.

From the local and extended sensitivity analysis (figure 3), we quantified the impact of each intervention component. For all the intervention components involved, the Ct prevalences have linear responses to the variation of each intervention parameter. The sensitivity indices quantify the impact and show that the two most significant intervention parameters are (1) the coverage of venue-based screening for men, which identifies and treats the Ct-positive men, and (2) the coverage of expedited partner treatment, which prevents the potential reinfection between the couple. Figure 4 shows the impact of partner treatment on the Ct prevalences but with much higher coverage of male-screening among the target population (increase from $7.5 \%$ to $40 \%$ ). The larger magnitude in the sensitivity index implies that the expedited partner treatment will be much more effective in reducing Ct prevalence when increasing the screening coverage in men.

By using the global sensitivity analysis on these two most significant intervention parameters, the model further explores the potential impact on a larger parameters space. Figure 5 gives the prediction of the Ct prevalences under different combinations of intervention parameters. At the current Check It intervention level, the impact could be limited by the resources and the capacity of the programme and the policy imposed on the protocol. In the adapted Check It protocol (as of February 2020), the expedited partner treatment incorporated the practices of patient delivered partner therapy and mail delivery, which improved the fraction of partner treatment from $27 \%$ to nearly $40 \%$. Moreover, if male-screening could be part of the standard recommendation as it is for women and if a moderate compliance rate of $30 \%$ could be achieved, then the model predicts that this joint screening programme will result in 30\% relative reduction in the Ct prevalence for the AA young women. After three decades of routinely screening women with no improvement in Ct 
rates, alternative interventions must be considered. This model provides support for changing the recommendations to include routine screening for men in addition to the current recommendations and best Ct control practices like EPT.

Our model considered a comprehensive picture of the current Ct epidemic in a young AA community and the control strategies implemented in men and women, separately. The model is parametrised using the survey data from the target population in New Orleans. This allows a detailed reconstruction of the assortative sexual mixing among the population and gives realistic predictions on the spread of the Ct epidemic and assessments on the mitigation efforts.

Although the model is parametrised based on the data sets from New Orleans, they represent similar cities that have high Ct rates (urban, southern and largely impoverished AA community) in many ways. Our study presented a robust and flexible model framework that could be adapted for the Ct epidemics in other similar populations. Nevertheless, the quantitative results presented in this study can only be interpreted for other cities after carefully examining the differences between sexual behaviour and assortative mixing patterns in two populations.

Many of our model limitations are associated with the scope of the available data sets. The uncertainty and bias in the model parameters and model assumptions can affect the reliability of the quantitative predictions. For example, due to the ethical challenges, rigorous estimates from longitudinal studies on the natural history of chlamydia are not available. ${ }^{20}$ We have assumed the same natural recovery period for men and women, and we studied the impact of this uncertainty in the (online supplemental Appendix A.5). Our assumptions on the partner updating frequency were made due to a lack of longitudinal data on the subject. Still, our numerical simulations (in online supplemental appendix A.1.3) suggested that, on the model recalibration, the relative ranking of the responses in the sensitivity analysis and the trend in prediction pattern is robust and insensitive. Also, Check It is still an ongoing study, and some of our model parameters may be further refined using the incoming data.

The dynamic sexual networks approximated the sexual behaviour between the high-risk age cohorts from Check It and YGG studies (men 15 to 24 and women 18 to 19). Meanwhile, we noticed a minority of women $(6 \%)$ in the YGG study reported partners from different age cohort ( $>24$ years old). These partnerships, which may serve as a residual reservoir, could not be detected by Check It programme that targets age range 15 to 24 . However, due to the lack of further data on quantifying these sexual behaviours, we did not include the intergenerational partnership with large age differences.

In the current study, we did not incorporate the demographic characteristics of the individual. This may lead to some bias in modelling the mixing (eg, age-mixing) structure for the sexual partnership. We reduced this bias by embedding the sexual networks into a grand social network, which considers an individual's age, ethnicity, social groups, economic status and geographical location. When updating the sexual networks, people will be more likely to establish sexual partnerships with their frequent social contacts. Another model assumption due to the lack of age is we didn't include the ageing effect: people will not be removed as they get older than eligible age range, and no new susceptible young people will come into the population. Thus, the caveat is the model will only be a good approximation for a limited time, and it is not suitable for simulations over a long period. Our future modelling study will be focussed on improving the model, such as considering the ageing process in the population and accounting for sexual partnerships outside the current range. These improvements will help us better quantify the impact of a male-screening programme.

We recognise that mathematical models are a simplistic representation of the real world. The quantitative predictions from our model may need further validation using incoming data stream on Ct prevalences, however, the qualitative results of our analysis adds to the evidence that male-screening interventions, together with other best practices in Ct control, has the potential to further mitigate the Ct prevalence among women in a high-prevalence community. Our future work will include further model calibration and validation using the incoming data from the Check It programme and evaluating the costeffectiveness of the male-screening programme.

\section{Author affiliations}

${ }^{1}$ Department of Mathematics, Tulane University, New Orleans, Louisiana, USA ${ }^{2}$ Department of Mathematics, The University of Texas at San Antonio, San Antonio, Texas, USA

${ }^{3}$ Simon A Levin Mathematical Computational Modeling Science Center, Arizona State University, Tempe, Arizona, USA

${ }^{4}$ Department of Epidemiology, Tulane University, New Orleans, Louisiana, USA ${ }^{5}$ Department of Health Policy and Management, Tulane University, New Orleans, Louisiana, USA

Acknowledgements We thank William Geisler (University of Alabama at Birmingham) for his helpful insight into the natural history of Chlamydia in men and women. We are also grateful to M Jacques Nsuami (Louisiana State University Health Sciences Center) whose comments and suggestions helped improve and clarify this manuscript.

Contributors All authors meet the ICMJE criteria for authorship. ZQ, AA and JH contributed to the design of the mathematical model. ZQ conducted data analysis, undertook numerical simulations and visualisation and interpreted results. NS and MCC-K contributed to data collection and analysis and reviewed model design and interpretation. CS reviewed the model design and interpretation. JH and PK contributed to the study, reviewed the model, data analysis and results interpretations and oversaw and coordinated the investigation. ZQ wrote the first draft of the article. All authors contributed to the writing and review of the draft and approved final manuscripts.

Funding This work was supported by the grants from the National Institutes of Health National Institute of Child Health and Human Development (R01HD086794) and the endowment for the Evelyn and John G Phillips Distinguished Chair in Mathematics at Tulane University. The content is solely the responsibility of the authors and does not necessarily represent the official views of the National Institutes of Health.

Competing interests None declared.

Patient consent for publication Not required. 
Ethics approval Tulane University Institutional Review Board.

Provenance and peer review Not commissioned; externally peer reviewed.

Data availability statement No data are available.

Supplemental material This content has been supplied by the author(s). It has not been vetted by BMJ Publishing Group Limited (BMJ) and may not have been peer-reviewed. Any opinions or recommendations discussed are solely those of the author(s) and are not endorsed by BMJ. BMJ disclaims all liability and responsibility arising from any reliance placed on the content. Where the content includes any translated material, BMJ does not warrant the accuracy and reliability of the translations (including but not limited to local regulations, clinical guidelines, terminology, drug names and drug dosages), and is not responsible for any error and/or omissions arising from translation and adaptation or otherwise.

Open access This is an open access article distributed in accordance with the Creative Commons Attribution Non Commercial (CC BY-NC 4.0) license, which permits others to distribute, remix, adapt, build upon this work non-commercially, and license their derivative works on different terms, provided the original work is properly cited, appropriate credit is given, any changes made indicated, and the use is non-commercial. See: http://creativecommons.org/licenses/by-nc/4.0/.

\section{ORCID iDs}

Zhuolin Qu http://orcid.org/0000-0002-8653-5975

Patricia J Kissinger http://orcid.org/0000-0002-2038-9151

\section{REFERENCES}

1 Centers for Disease Control and Prevention. Sexually transmitted disease surveillance 2018. Atlanta US Dep Health Hum Serv 2019.

2 Hillis SD, Wasserheit JN. Screening for chlamydia--a key to the prevention of pelvic inflammatory disease. $N$ Engl J Med 1996;334:1399-401.

3 Ward H, Rönn M. Contribution of sexually transmitted infections to the sexual transmission of HIV. Curr Opin HIV AIDS 2010;5:305-10.

4 Centers for Disease Control and Prevention. Male Chlamydia screening consultation, March 28 - 29, 2006, meeting report. Atlanta, Georgia, 2007. Available: https://www.cdc.gov/std/chlamydia/ chlamydiascreening-males.pdf

5 Gift TL, Gaydos CA, Kent CK, et al. The program cost and costeffectiveness of screening men for Chlamydia to prevent pelvic inflammatory disease in women. Sex Transm Dis 2008;35:S66-75.

6 Gopalappa C, Huang Y-LA, Gift TL, et al. Cost-Effectiveness of screening men in Maricopa County jails for Chlamydia and gonorrhea to avert infections in women. Sex Transm Dis 2013;40:776-83.

7 Kissinger P, Schmidt N, Gomes G. 014.2 can community Chlamydia trachomatis screening of young heterosexual men help identify infected networks? Sex Transm Infect 2019;95:A71

8 Green J, Schmidt N, Latimer J. WP 58 the influence of partnership type and characteristics on condom use among young African American women. Sex Transm Dis 2014;41:S111

9 Network Dynamics and Simulation and Science Laboratory. Synthetic data products for societal infrastructures and protopopulations: data set 2.0. Virginia Polytechnic Institute and state university, 2008. Available: http://ndssl.vbi.vt.edu/Publications/ ndssl-tr-07-003.pdf

10 Farley TA, Cohen DA, Elkins W. Asymptomatic sexually transmitted diseases: the case for screening. Prev Med 2003;36:502-9.

11 Centers for Disease Control and Prevention. Sexually transmitted diseases (STDs), Expedited partner therapy, 2019. Available: https:// www.cdc.gov/std/ept/default.htm [Accessed 21 Apr 2020].
12 Centers for Disease Control and Prevention. Chlamydia treatment and care, 2016. Available: https://www.cdc.gov/std/chlamydia/ treatment.htm [Accessed 21 Apr 2020].

13 St Lawrence JS, Montaño DE, Kasprzyk D, et al. Std screening, testing, case reporting, and clinical and partner notification practices: a national survey of US physicians. Am J Public Health 2002;92:1784-8.

14 Quinn TC, Gaydos C, Shepherd M, et al. Epidemiologic and microbiologic correlates of Chlamydia trachomatis infection in sexual partnerships. JAMA 1996;276:1737-42.

15 Kretzschmar M, van Duynhoven YT, Severijnen AJ. Modeling prevention strategies for gonorrhea and Chlamydia using stochastic network simulations. Am J Epidemiol 1996;144:306-17.

16 Turner KME, Adams EJ, Gay N, et al. Developing a realistic sexual network model of Chlamydia transmission in Britain. Theor Biol Med Model 2006;3:3.

17 Torrone E, Papp J, Weinstock H, et al. Prevalence of Chlamydia trachomatis genital infection among persons aged 14-39 years-United States, 2007-2012. MMWR Morb Mortal Wkly Rep 2014;63:834-8.

18 Molano M, Meijer CJLM, Weiderpass E, et al. The natural course of Chlamydia trachomatis infection in asymptomatic Colombian women: a 5-year follow-up study. J Infect Dis 2005;191:907-16.

19 Chitnis N, Hyman JM, Cushing JM. Determining important parameters in the spread of malaria through the sensitivity analysis of a mathematical model. Bull Math Biol 2008;70:1272-96.

20 Geisler WM, untreated Dof. Duration of untreated, uncomplicated Chlamydia trachomatis genital infection and factors associated with Chlamydia resolution: a review of human studies. J Infect Dis 2010;201 Suppl 2:104-13.

21 Martin DH, Mroczkowski TF, Dalu ZA, et al. A controlled trial of a single dose of azithromycin for the treatment of chlamydial urethritis and cervicitis. N Engl J Med 1992;327:921-5.

22 Trussell J, Guthrie K. Choosing a contraceptive: efficacy, safety, and personal considerations. In: Contraceptive technology. 19th edn. New York (NY: Ardent Media, Inc, 2007: 19-47.

23 Centers for Disease Control and Prevention. Chlamydia, detailed fact sheet, 2016. Available: https://www.cdc.gov/std/chlamydia/stdfactchlamydia-detailed.htm [Accessed 21 Apr 2020].

24 Wiehe SE, Rosenman MB, Wang J, et al. Chlamydia screening among young women: individual- and provider-level differences in testing. Pediatrics 2011;127:e336-44.

25 Hoover KW, Leichliter JS, Torrone EA, et al. Chlamydia screening among females aged 15-21 years--multiple data sources, United States, 1999-2010. MMWR Supp/ 2014;63:80-8.

26 Hogben M, McCree DH, Golden MR. Patient-Delivered partner therapy for sexually transmitted diseases as practiced by U.S. physicians. Sex Transm Dis 2005;32:101-5.

27 Golden MR, Whittington WLH, Handsfield HH, et al. Effect of expedited treatment of sex partners on recurrent or persistent gonorrhea or chlamydial infection. N Engl J Med 2005;352:676-85.

28 Schillinger JA, Kissinger $\mathrm{P}$, Calvet $\mathrm{H}$, et al. Patient-Delivered partner treatment with azithromycin to prevent repeated Chlamydia trachomatis infection among women: a randomized, controlled trial. Sex Transm Dis 2003;30:49-56.

29 Xu F, Stoner BP, Taylor SN, et al. Use of home-obtained vaginal swabs to facilitate rescreening for Chlamydia trachomatis infections: two randomized controlled trials. Obstet Gynecol 2011;118:231-9.

30 RelayHealth. gonorrhea and Chlamydia tests, 2014. Available: https://www.summitmedicalgroup.com/library/adult_health/aha gonorrhea_and_chlamydia_tests [Accessed 2 Feb 2020]. 